\title{
Endoscopic and Histological Patchiness in Treated Ulcerative Colitis
}

\author{
Byungki Kim, M.D., Jeffrey L. Barnett, M.D., Celina G. Kleer, M.D., and Henry D. Appelman, M.D. \\ Division of Gastroenterology and Department of Pathology, University of Michigan Medical Center, \\ Ann Arbor, Michigan
}

OBJECTIVE: Traditionally, contiguous distribution of inflammation (endoscopic and histological) with rectal involvement is thought to be important in distinguishing ulcerative colitis (UC) from Crohn's disease of the colon. Little longterm data are available that prove whether this rule holds during the course of disease as it is modified by time and treatment. The aim of this study was to investigate the prevalence of endoscopic and histological patchiness and rectal sparing in treated UC over time and to correlate these findings with treatment at the time of endoscopy.

METHODS: Patients with well-established UC who underwent sequential colonoscopy or flexible sigmoidoscopy with biopsies were included in this study. Patients' medical records including endoscopy/biopsy reports and clinical status/symptoms/treatment at the time of endoscopy were reviewed retrospectively.

RESULTS: A total of 32 patients (14 men, 18 women; median age, $45 \mathrm{yr}$; median UC duration, $15 \mathrm{yr}$ ) underwent 175 sequential endoscopies with biopsies (161 colonoscopies, 14 sigmoidoscopies; median, five endoscopies per patient; range, 3-10). Endoscopic and/or histological patchiness was present in 20 of $175(11 \%)$ sequential endoscopies with biopsies over time from 12 of $32(38 \%)$ patients. Endoscopic and/or histological rectal sparing was present in 27 of $175(15 \%)$ of sequential endoscopies with biopsies over time from 14 of 32 (44\%) patients. Seven patients had both patchiness and rectal sparing. Therefore, in 47 (27\%) follow-up endoscopies in 19 (59\%) patients, there was either patchy disease, rectal sparing, or both sometime during the course of disease with treatment. There was no significant difference in treatment, including steroid use and rectal therapy, between those with patchiness and/or rectal sparing and those without.

CONCLUSIONS: Contrary to traditional teaching, endoscopic and histological patchiness of inflammation and rectal sparing are common during the course of disease in treated UC and seem to be unrelated to specific therapy. (Am J Gastroenterol 1999;94:3258-3262. (C) 1999 by Am. Coll. of Gastroenterology)

The results of this study were presented at the American College of Gastroenterology 63rd Annual Scientific Meeting, Boston, Massachusetts, October 1998.

\section{INTRODUCTION}

Ulcerative colitis and Crohn's disease are differentiated by a set of clinical, endoscopic, and histological characteristics (1). There is no single endoscopic or histological finding that is absolutely diagnostic of one disease or the other. One of the characteristics that is believed to be important in distinguishing ulcerative colitis from Crohn's disease is the pattern of endoscopic and histological distribution of inflammation. According to traditional teaching, ulcerative colitis involves contiguous areas of the colon without skip lesions, starting from the rectum (2-7). The presence of patchy inflammation or rectal sparing in a patient with previously diagnosed ulcerative colitis may lead to a change of diagnosis to Crohn's disease. Such a change in diagnosis may have a significant impact on management strategies. For instance, a patient with intractable ulcerative colitis who is found to have patchy involvement or rectal sparing may not be considered a candidate for curative colectomy with ileoanal pouch procedure because these distributions suggest the possibility of Crohn's disease.

To date, little long-term data are available on whether the traditional teaching holds during the course of disease with treatment. There have been a few studies that refute the dogma regarding the classic distribution of inflammation in treated ulcerative colitis (8-13). In some of these studies, however, patients usually had one endoscopy with biopsy and were not followed further with subsequent endoscopy. Also, these studies adopted less strict definitions of patchiness and rectal sparing and included cases with relative patchiness or rectal sparing based on endoscopic and histological differences in the severity of inflammation. One recent study has suggested that the extent and the pattern of distribution of inflammation in chronic ulcerative colitis may vary significantly after $1 \mathrm{yr}$ of treatment (14). Our previous study was the first to investigate the prevalence of histological patchiness or rectal sparing in patients with treated ulcerative colitis over time based upon sequential endoscopies with biopsies (13). In this study, we expand upon that report by providing a detailed endoscopic and clinical arm and correlating it with histological data to describe patchiness and rectal sparing in patients with wellestablished ulcerative colitis who underwent sequential endoscopies with biopsies over as long a period as $13 \mathrm{yr}$. 


\section{MATERIALS AND METHODS}

Patients with well-documented ulcerative colitis followed at the University of Michigan Medical Center Gastroenterology Clinic who underwent sequential colonoscopy or flexible sigmoidoscopy with biopsies for dysplasia surveillance or for disease flare were included in this study. Only patients with at least three separate sets of biopsies over at least a 2 -yr period were included. The patients were selected as they were identified during regular biopsy diagnostic signout in our pathology department. The diagnosis of ulcerative colitis was established by widely accepted clinical, endoscopic, and histological criteria $(6,7,15,16)$.

The patients' medical records were reviewed retrospectively. Patient data reviewed included demographics, duration of disease, extent and severity of disease at the time of diagnosis, endoscopy/biopsy findings, and patients' clinical status at the time of endoscopy/biopsy (flare, settling of flare, or remission). Clinical parameters reviewed included stool consistency and number of bowel movements per day, presence or absence of hematochezia, and treatment and response at the time of the procedure. Endoscopy/biopsy findings were considered to reflect steroid use and rectal therapy if these were used within 1 month of the procedure.

All biopsy slides were reviewed again by experienced gastrointestinal (GI) pathologists. Histological details including representative photographs have been recently published (13). Each colorectal biopsy was classified as definite or suspicious for chronic colitis, or normal. The biopsy was classified as definite chronic colitis when there was an obvious expansion of the lamina propria by plasma cells and/or eosinophils, and/or accompanying mucosal distortion. It was classified as suspicious for chronic colitis when there was minimal distortion without lamina propria inflammation, or if there were scattered metaplastic Paneth cells in the left colon or rectum without mucosal distortion or lamina propria inflammation.

Patchiness was defined as areas of normal mucosa (endoscopically and/or histologically) amid areas of apparent inflammation. Rectal sparing was defined as a normal rectum (endoscopically and/or histologically) when definite proximal disease was present. Relative patchiness and rectal sparing based on anatomic regional differences in the severity of inflammation were not included in this study.

Endoscopy and biopsy reports were compared, in terms of extent and severity of disease and the presence or absence of patchiness and/or rectal sparing. The endoscopic severity of disease was graded as mild, moderate, or severe by impressions on endoscopy reports based on widely accepted endoscopic criteria (1). The histological severity of disease was graded as mild, moderate, or severe based on the intensity of chronic inflammation (plasma cells, eosinophils), degree of mucosal distortion, and amount of neutrophil activity. Endoscopic and histological correlation, in terms of severity, was considered to be absent only when
Table 1. Prevalence of Patchiness

\begin{tabular}{lcc}
\hline & $\begin{array}{c}\text { Number of } \\
\text { Patients }\end{array}$ & $\begin{array}{c}\text { Number of } \\
\text { Episodes }\end{array}$ \\
\hline Endoscopic only & 3 & 4 \\
Histological only & 5 & 8 \\
Endo/histo* & 3 & 4 \\
Endo and histo $\dagger$ & 1 & 4 \\
Total & $12 / 32(38 \%)$ & $20 / 175(11 \%)$ \\
\hline
\end{tabular}

* Combined endo/histo patchiness at the same time.

$\dagger$ Both endo and histo patchiness over time, but only one type on each occasion.

Endo $=$ endoscopic; histo $=$ histological.

endoscopic and histological impressions differed by at least two grades (normal $v s$ moderate to severe or mild $v s$ severe).

The data were analyzed using the $\chi^{2}$ test. Statistical significance was defined as a $p$ value $<0.05$.

\section{RESULTS}

Thirty-two patients were included in this study. There were 14 men and 18 women with a median age of $45 \mathrm{yr}$ (range, 18-83 yr). The median duration of disease was $15 \mathrm{yr}$, ranging from 4 to $39 \mathrm{yr}$. The initial extent of disease at the time of diagnosis included proctitis in two, proctosigmoiditis in five, so-called "distal colitis" in three, left-sided colitis in three, colitis from rectum to transverse colon in two, and pancolitis in seven patients; it was poorly described or not clearly classified in 10 patients. A total of 175 sequential endoscopies with biopsies (161 colonoscopies, 14 sigmoidoscopies; median of five endoscopies per patient; range, three to 10) were performed in these patients over as long as a 13 -yr period.

Endoscopic and/or histological patchiness was present in 20 of 175 (11\%) sequential endoscopies with biopsies over time from 12 of $32(38 \%)$ patients. In these 12 patients, six endoscopic, 10 histological, and four combined endoscopic/ histological episodes of patchiness were present (Table 1). Three patients had endoscopic patchiness only: two patients had one episode each and one patient had two episodes on two different occasions. Five patients had histological patchiness only: four patients had one episode each and one patient had four episodes on four different occasions. Three patients had both endoscopic and histological patchiness at the same time: two patients had one episode each and one patient had two episodes on two different occasions. One patient had both endoscopic and histological patchiness over time, but only one type on each occasion: two endoscopic and two histological episodes on four different occasions.

Endoscopic and/or histological rectal sparing was present in 27 of $175(15 \%)$ sequential endoscopies with biopsies over time in 14 of 32 (44\%) patients. In these 14 patients, 15 endoscopic, 10 histological, and two combined endoscopic/ histological episodes of rectal sparing were present (Table 2). Five patients had endoscopic rectal sparing only: four patients had one episode each and one patient had three episodes on three different occasions. Four patients had 
Table 2. Prevalence of Rectal Sparing

\begin{tabular}{lcc}
\hline & $\begin{array}{c}\text { Number of } \\
\text { Patients }\end{array}$ & $\begin{array}{c}\text { Number of } \\
\text { Episodes }\end{array}$ \\
\hline Endoscopic only & 5 & 7 \\
Histological only & 4 & 6 \\
Endo/histo* & 1 & 1 \\
Endo and histo $\dagger$ & 4 & 13 \\
Total & $14 / 32(44 \%)$ & $27 / 175(15 \%)$ \\
\hline
\end{tabular}

* Combined endo/histo rectal sparing at the same time.

$\dagger$ Both endo and histo rectal sparing over time but only one type on each occasion.

Endo $=$ endoscopic; histo $=$ histological.

histological rectal sparing only: two patients had one episode each and two patients had two episodes on two different occasions. One patient had both endoscopic and histological rectal sparing at the same time on one occasion. Three patients had both endoscopic and histological rectal sparing over time, but only one type on each occasion: one patient had three endoscopic and one histological episodes on four different occasions, one patient had two endoscopic and one histological episodes on three different occasions, and one patient had one endoscopic and one histological episodes on two different occasions. One patient had endoscopic, histological, and endoscopic/histological rectal sparing over time (only one type on each occasion): two endoscopic, one histological, and one combined endoscopic/ histological episodes on four different occasions.

Seven patients had both patchiness and rectal sparing. Therefore, in 19 patients (59\%), 47 episodes (27\%) of endoscopic and/or histological patchiness and/or rectal sparing were present some time during the course of disease with treatment.

The most common single treatment at the time of the procedures was sulfasalazine in all groups of patients (Table $3)$. Seven episodes in the group with patchiness (35\%), seven episodes in the group with rectal sparing (26\%), and 25 episodes in the group without patchiness or rectal sparing (20\%) were treated with sulfasalazine alone at the time of the procedure. Three episodes $(15 \%)$ in the group with patchiness and eight episodes (30\%) in the group with rectal sparing, and 30 episodes (23\%) in the group without patchiness or rectal sparing were treated with a steroid, usually in combination with other medications. Five episodes (18\%) in the group with rectal sparing, two episodes in the group with patchiness $(10 \%)$, and 17 episodes $(13 \%)$ in the group

Table 3. Treatment at the Time of Endoscopy (Number of Episodes)

\begin{tabular}{lccc}
\hline & $\begin{array}{c}\text { Group With } \\
\text { Patchiness } \\
(\mathrm{N}=20)\end{array}$ & $\begin{array}{c}\text { Group With } \\
\text { Rectal Sparing } \\
(\mathrm{N}=27)\end{array}$ & $\begin{array}{c}\text { Group Without } \\
\text { Patchiness/ } \\
\text { Rectal Sparing } \\
(\mathrm{N}=128)\end{array}$ \\
\hline Sulfasalazine & $7(35 \%)$ & $7(26 \%)$ & $25(20 \%)$ \\
Steroid* & $3(15 \%)$ & $8(30 \%)$ & $30(23 \%)$ \\
Rectal therapy & $2(10 \%)$ & $5(18 \%)$ & $17(13 \%)$ \\
\hline
\end{tabular}

* Most patients were treated with a steroid in combination with other medications, such as mesalamine and sulfasalazine. without rectal sparing or patchiness were treated with rectal therapy (either steroid or mesalamine enemas).

In 90 episodes $(51 \%)$ there was lack of endoscopic and histological correlation. The lack of correlation was based on the extent of disease in 52 episodes, on severity in seven, and on both the extent of disease and severity in 11 episodes; in 17 episodes endoscopy was normal but histological evidence of at least moderate colitis was present; in three episodes, histology was normal but endoscopic evidence of colitis was present.

Endoscopic and/or histological disease reverted to normal in $23(13 \%)$ episodes in 12 patients (38\%) some time during the course of disease. These patients had endoscopic and/or histological evidence of colitis on subsequent endoscopy(ies).

None of the patients with patchiness and/or rectal sparing developed any symptoms or complications suggestive of Crohn's disease during the study period, and their diagnosis of ulcerative colitis remained unchanged.

\section{DISCUSSION}

To the best of our knowledge, this is the first study that investigated the prevalence of both endoscopic and histological patchiness and rectal sparing in patients with treated ulcerative colitis over time with sequential endoscopic biopsies. Also, in this study patchiness and rectal sparing were defined in absolute terms (present or absent), and cases with relative patchiness and/or rectal sparing were not included.

Contrary to traditional teaching on the classic distribution of inflammation in ulcerative colitis, our data show that endoscopic and/or histological patchiness and rectal sparing are not uncommon findings in patients with longstanding treated ulcerative colitis over time. Nineteen of 32 patients (59\%) with well-documented ulcerative colitis in our study had patchiness and/or rectal sparing (endoscopic and/or histological) some time during the course of their disease. Twelve patients (38\%) had 20 episodes of patchiness, and 14 patients (44\%) had 27 episodes of rectal sparing. The episodes of patchiness and rectal sparing were found endoscopically and histologically in an approximately equal distribution.

Our results are supported by the findings of previous studies that challenged the dogma regarding the classic distribution of inflammation in ulcerative colitis $(8-12,17)$. A prospective study by Bernstein et al. showed that of 39 patients with well-established ulcerative colitis, 17 patients (44\%) had endoscopic and 13 patients (33\%) had histological patchiness (including five with endoscopic and six with histological rectal sparing), in absolute or relative terms (8). Absolute patchiness or rectal sparing was present in five patients (13\%): three with endoscopic patchiness and two with histological rectal sparing. In this study, the patients had only one endoscopy with biopsies and were not followed further with subsequent endoscopy. We adopted a strict definition of patchiness and rectal sparing and ex- 
cluded cases with relative disease. The prevalence of absolute patchiness/rectal sparing in our study of $59 \%$ of the patients is clearly higher than the $13 \%$ prevalence identified in the Bernstein series. This difference is probably due to the long-term endoscopic follow-up in our patients and suggests that with repeated biopsies over the course of years, findings of patchiness/rectal sparing are not only possible but perhaps are to be expected.

D'Haens et al., in a prospective study employing complete ileocolonoscopy on 20 patients with established leftsided ulcerative colitis, found a high prevalence of isolated proximal patchy inflammation (9). Fifteen patients (75\%) had a patch of inflammation (both endoscopic and histological) in the cecum. In all cases this patch of inflammation was separated from the obviously inflamed distal segment by normal mucosa or mucosa with only borderline changes (endoscopically and/or histologically); the terminal ileum appeared normal in all cases. Upon follow-up of these patients for at least $8 \mathrm{yr}$, none developed ileitis or anal complications suggestive of Crohn's disease, and their diagnosis remained unchanged. Interestingly, all 15 patients had obvious patchy inflammation in the periappendiceal area of the cecum with a normal or relatively normal ascending and transverse colon. The significance of this pattern of disease involvement in these patients indicates that skip lesions do occur in ulcerative colitis, a challenge to conventional dogma about the distribution of changes in ulcerative colitis as opposed to Crohn's disease. However, this is a different finding from the changing nature of treated disease with respect to the patchiness that we are describing.

The prevalence of rectal sparing in treated ulcerative colitis has been investigated in a few studies $(10-12,18)$. In a series of 12 patients who had been categorized as having ulcerative colitis with predominantly right-sided disease or relative rectal sparing, six (50\%) had a normal sigmoidoscopic appearance with evidence of proximal disease on double-contrast barium enema (18). In these patients, however, rectal biopsies showed changes compatible with ulcerative colitis (three with only mild changes). Oshitani et al. reported nine of 30 patients (30\%) with ulcerative colitis who had absolute or relative endoscopic rectal sparing; however, no biopsies were taken in this study (11). In 14 patients enrolled in a topical 5-aminosalicylic acid treatment trial, nine patients $(64 \%)$ were later found to have normal rectal biopsies, although, interestingly, two had been treated with placebo (12). More recently, Levine et al. found that 11 of 24 patients $(46 \%)$ with well-documented ulcerative colitis had normal or only borderline abnormal rectal biopsies (two with normal biopsies); they did not describe endoscopic findings (10). These studies were limited to rectal sparing, and except for one study, did not attempt to correlate endoscopic findings with histological findings (18).

Finally, in untreated ulcerative colitis, patchy inflammation and rectal sparing have been described in children. Markowitz et al. reported that five of 12 children with proven ulcerative colitis by examination of a subtotal co- lectomy specimen had either mild patchy inflammation or rectal sparing on biopsies at their disease onset (19). It is not clear whether these data can be extrapolated to adult patients, but it suggests the possibility that patchy inflammation and rectal sparing can occur even in patients with untreated ulcerative colitis.

In our study, there was no significant difference in treatment between those with patchiness and/or rectal sparing and those without. The most common single treatment in all groups of patients was sulfasalazine. Thirty-five percent of episodes with patchiness, $26 \%$ of episodes with rectal sparing, and $20 \%$ of episodes without patchiness or rectal sparing were treated with sulfasalazine alone at the time of procedure; the difference was not statistically significant. There was also no difference between patients with patchiness and/or rectal sparing and those without based upon the use of oral steroids. Even topical therapy appeared to have little influence on rectal histology in our ulcerative colitis patients. We found that $18 \%$ of episodes with rectal sparing and $13 \%$ of episodes without rectal sparing were treated with topical rectal therapy, either mesalamine or steroid enemas $(p=0.43)$. Previously mentioned studies also showed no difference in the use of enemas between patients with rectal sparing and those without $(8,11)$. In the prospective mesalamine enema treatment trial discussed earlier, the patients treated with mesalamine enemas had a higher average number of normal rectal biopsies per patient than those treated with placebo enemas (3 vs 1.3) (12). However, these were patients with active left-sided colitis on a treatment protocol and our patients were biopsied during varying degrees of disease activity and treatment. Under chronic conditions, the use of medication at the time endoscopy with biopsy is performed appears to have little influence on the presence of patchiness and rectal sparing.

A recent study by Moum et al. suggested that the discrepancy between endoscopic and histological involvement in ulcerative colitis patients increases with time (14). In this study, histopathological examination showed more extensive disease than endoscopic findings in $4 \%$ at diagnosis and in $28 \%$ at follow-up in $1 \mathrm{yr}$, whereas endoscopic findings showed more extensive disease than histopathological examination in $18 \%$ at diagnosis and $12 \%$ at follow-up. Our results show lack of correlation between endoscopic and histological findings in $51 \%$ of episodes. Evidence of histological disease was present in 17 episodes with endoscopically normal mucosa, and histologically normal mucosa appeared abnormal endoscopically in three episodes. Thus, our results suggest that lack of endoscopic and histological correlation is not uncommon in patients with treated longterm ulcerative colitis. To assess the maximal extent and severity of disease in patients with ulcerative colitis, biopsies should be taken from normal-appearing as well as abnormal-appearing mucosae, and both endoscopic and histological findings should be considered.

In summary, our results defy the traditional rule requiring the presence of contiguous distribution of inflammation with 
rectal involvement in ulcerative colitis. Patchy distribution of inflammation and rectal sparing are common in patients with treated ulcerative colitis, and do not seem to be related to any specific treatment, including steroid use and rectal therapy. Our results support and extend the findings of a few previous studies by so-called "revisionists" $(8-12)$. The finding of patchy inflammation and/or rectal sparing alone in patients with previously diagnosed ulcerative colitis should not necessarily lead to a change of diagnosis to Crohn's disease; comprehensive evaluation including careful review of previous biopsies should be done before raising the suspicion of Crohn's disease. In patients with ulcerative colitis, the extent and the pattern of distribution of inflammation may vary significantly over time with treatment, including reversion to normal mucosa, and lack of endoscopic and histological correlation appears to be relatively common.

Reprint requests and correspondence: Jeffrey L. Barnett, M.D., University of Michigan Medical Center, 1500 East Medical Center Drive, UH2A341, Ann Arbor, MI 48109-0051.

Received Jan. 28, 1999; accepted May 11, 1999.

\section{REFERENCES}

1. Stenson W. Inflammatory bowel disease. In: Yamada T, Alpers DH, Owyang C, eds. Textbook of gastroenterology, 2nd edition. Philadelphia: JB Lippincott Co., 1995:1748-72.

2. Hogan WJ, Hensley GT, Geenen JE. Endoscopic evaluation of inflammatory bowel disease. Med Clin North Am 1980;64: 1083-1102.

3. Waye J. The role of colonoscopy in the differential diagnosis of inflammatory bowel disease. Gastrointest Endosc 1977;23: $150-4$.

4. Donnelan WL. Early histological changes in ulcerative colitis. Gastroenterology 1966;50:519-39.

5. Haggitt RC. Ulcerative colitis. In: Goldman H, Appelman HD, Kauffman N, eds. Gastrointestinal pathology. Baltimore: Williams \& Wilkins, 1990: 325-55.

6. Lewin KJ, Riddell RH, Weinstein WM. Inflammatory bowel diseases. In: Lewin KJ, Riddell RH, Weinstein WM, eds. Gastrointestinal pathology and its clinical implications. New York: Igaku-Shoin, 1992:838-58.

7. Goldman H. Ulcerative colitis and Crohn's disease. In: Ming S, Goldman H, eds. Pathology of the gastrointestinal tract. Philadelphia. WB Saunders, 1992:643-76.

8. Bernstein CN, Shanahan F, Anton P, et al. Patchiness of mucosal inflammation in treated ulcerative colitis. A prospective study. Gastrointest Endosc 1995;42:232-7.

9. D'Haens G, Geboes K, Peeters M, et al. Patchy cecal inflammation associated with distal ulcerative colitis. A prospective endoscopic study. Am J Gastroenterol 1997;92:1275-9.

10. Levine TS, Tzardi M, Mitchell S, et al. Diagnostic difficulty arising from rectal recovery in ulcerative colitis. J Clin Pathol 1996;49:319-23.

11. Oshitani N, Kitano A, Nakamura S, et al. Clinical and prognostic features of rectal sparing in ulcerative colitis. Digestion 1989;42:39-43.

12. Odze R, Antonioli D, Peppercorn M, et al. Effect of topical 5-aminosalicylic acid (5-ASA) treatment on rectal mucosal biopsy morphology in chronic ulcerative colitis. Am J Surg Pathol 1993;17:869-75.

13. Kleer CG, Appelman HD. Ulcerative colitis: Patterns of involvement in colorectal biopsies and changes with time. Am J Surg Pathol 1998;22:983-9.

14. Moum B, Vatn M, Ekbom A, et al. Endoscopic and histological evaluation of extent of disease in ulcerative colitis: Differences increase from diagnosis, and until follow-up one year later. Gastroenterology 1997;112:A-1090 (abstract).

15. Geller S. Pathology of inflammatory bowel disease. A critical appraisal in diagnosis, and management. In: Targan SR, Shanahan F, eds. Inflammatory bowel disease, from bench to bedside. Baltimore: Williams \& Wilkins, 1994: 336-51.

16. Sutherland LR. Clinical course and complications of ulcerative colitis, and ulcerative proctitis. In: Targan SR, Shanahan $\mathrm{F}$, eds. Inflammatory bowel disease, from bench to bedside. Baltimore: Williams \& Wilkins, 194:279-93.

17. Bernstein $\mathrm{CN}$. On making the diagnosis of ulcerative colitis. Editorial. Am J Gastroenterol 1997;8:1247-52.

18. Spiliadis CA, Spiliadis CA, Lennard-Jones JE. Ulcerative colitis with relative sparing of rectum. Clinical features, histology, and prognosis. Dis Colon Rectum 1987;30:334-6.

19. Markowitz J, Kahn E, Grancher K, et al. Atypical rectosigmoid histology in children with newly diagnosed ulcerative colitis. Am J Gastroenterol 1993;88:2034-7. 